\title{
Ginzburg Criterion for the Chiral Transition
}

\author{
M.A. Stephanov ${ }^{\mathrm{a}}$ \\ anstitute for Theoretical Physics, SUNY, Stony Brook, NY 11794-3840
}

This report is based on the work done together with J.B. Kogut and C.G. Strouthos. We study a Yukawa theory with spontaneous chiral symmetry breaking and with a large number $N$ of fermions near the finite temperature phase transition. Critical properties in such a system can be described by the mean field theory very close to the transition point. We show that the width of the window of non-trivial scaling is suppressed by a certain power of $1 /$ N. Our Monte Carlo simulations confirm these analytical results. We discuss implications for the chiral phase transition in QCD.

\section{Introduction}

Global symmetries provide important information about the properties of quantum field theories. In $\mathrm{QCD}$ with 2 massless quarks the global symmetries form the group: $\mathrm{SU}_{A}(2) \times \mathrm{SU}(2)_{V}$ $\times \mathrm{U}_{B}(1)$. This fact, together with the ideas of dimensional reduction and universality gives us a prediction for the long-scale behavior at the chiral symmetry restoration transition in QCD [1].

The idea of dimensional reduction is based on the observation that at the second order phase transition a certain correlation length in the system diverges and eventually becomes longer than the extent of the Euclidean time. The longwavelength fluctuations are then squeezed to a $d-1$ dimensional "pancake". The universality tells us that the long-wavelength behavior of a system depends on the global symmetry and the dimensionality of the system. In particular, for the QCD with 2 massless quarks one should find $\mathrm{O}(4)=\mathrm{SU}(2) \times \mathrm{SU}(2)$ exponents at the chiral phase transition [1].

Such an argument, based on universality, does not tell us how this universal critical behavior sets in. To answer this question one has to study the dynamics. In this work we study how this universal behavior sets in for theories with large number, $N$, of fermion species.

The fermionic fields, unlike the bosonic, do not survive dimensional reduction. One way to see this is to recall that fermion fields have antiperiodic boundary conditions in the time di- rection, which suppress fluctuations when their correlation length exceeds $1 / T$. In another language, the effective $d$-1-dimensional theory for the bosonic fields near $T_{c}$ is a classical statistical theory. Fermion fields do not have a classical limit [2].

However, the fermions may affect the way that the universal behavior sets in. Several authors observed [3, 1 that in the large $N$ limit the exponents of the finite $T$ chiral transition in the Yukawa model are given by the mean field (MF) theory. Monte Carlo simulations confirmed this result [4. How does it reconcile with the universality arguments? The answer is that the large $N$ description has its region of applicability. The scaling for the correlation length in the window between $1 / T$ and $N^{x} / T, x>0$, is indeed given by MF. However, when the correlation length exceeds $N^{x} / T$ the universal scaling sets in.

\section{The model}

To derive the crossover exponent $x$ we study [5] a Yukawa theory with large number $N$ of fermion species. It can be thought of as an effective description of degrees of freedom in QCD which participate in chiral symmetry breaking. We choose the symmetry group $\mathrm{Z}(2)$ instead of $\mathrm{O}(4)$ to simplify the argument. The theory is defined by the Lagrangian:

$$
\mathcal{L}=\frac{1}{2}(\partial \phi)^{2}+\frac{1}{2} \mu^{2} \phi^{2}+\lambda \phi^{4}+\sum_{f=1}^{N} \bar{\psi}_{f}(\not \partial+g \phi) \psi_{f}(1)
$$


and is regularized by some momentum cutoff, $\Lambda$. There are two other important scales in the theory: the temperature, $T$, and the mass $m$ of the thermal excitations of the scalar field. $m$ vanishes at $T_{c}$. Therefore, near the finite temperature phase transition we have the following hierarchy of scales: $\Lambda \gg T \gg m$.

The renormalization group (RG) evolution of the quartic self-coupling of the scalar field, $\lambda$, from the scale $\Lambda$ to the scale $T$ is governed by the RG equations of the $d$-dimensional quantum Yukawa model. After that, at the scale of $T$, we pass through a crossover region: the fermions and nonzero Matsubara modes of the scalar fields decouple. The evolution below $T$ is governed by the RG equations of the scalar $\phi^{4}$ theory in $d-1$ dimensions.

If the window of scales between $\Lambda$ and $T$ is wide the renormalized coupling $\lambda$ at the scale $T$, $\lambda(T)$, is close to the infrared fixed point of the $d$ dimensional Yukawa theory. In the large- $N$ limit:

$\lambda(T) \sim \frac{(4-d) T^{4-d}}{N}$ for $2<d<4$.

The case $d=4$ is special. The infrared fixed point is trivial and is approached logarithmically as $\Lambda / T \rightarrow \infty: \lambda(T) \sim 1 /[N \ln (\Lambda / T)]$. For large $N$ this coupling is small. As we shall see shortly, this is the reason why the non-MF critical region is reached only very close to the phase transition.

The quantitative relation between the size of the non-MF critical region, the Ginzburg region, and certain parameters of a given system is known as the Ginzburg criterion. In superconductors such a parameter is a small ratio $T / E_{F}$, i.e., the width of the Ginzburg region is suppressed by a power of this parameter. In a field theory with large number of fermions, such as (1), such a parameter is $1 / N$.

The MF approximation breaks down because of self-inconsistency when the fluctuations become large. The size of the corrections to the MF is determined by the value of the effective self-coupling of the scalar field. Since the dimensionality of the coupling of the $d$-1-dimensional scalar theory, $\lambda_{d-1}=T \lambda(T)$, is $5-d$, the MF approximation breaks down when $\lambda_{d-1} \sim m^{5-d}$. From this, and with the help of (2), one obtains the following criterion for the applicability of the MF scaling:

$m \gg \frac{T}{N^{x}}, \quad x=\frac{1}{5-d}$.

In the special case of $d=4$ one finds: $m \gg$ $T /[N \ln (\Lambda / T)]$.

The Ginzburg criterion (3) says that for masses $m$ inside the window $T \gg m \gg T / N^{x}$ the MF scaling holds, while for smaller masses $m \ll$ $T / N^{x}$ (i.e., closer to the transition) the nontrivial $d-1$ Ising scaling sets in. We see that the size of this latter, non-trivial critical region is suppressed at large $N$.

\section{Lattice}

The strategy we use to confirm our analytical results is the following. First, we show that the behavior at sufficiently large correlation length is given by the universality arguments, which in this case predict Ising $Z(2)$ critical exponents. Second, we identify the boundary of the MF region, test the scaling and extract the exponent $x$.

We discretize the $d=3$ theory (1) on a cubic $L_{s}^{2} \times L_{t}$ lattice in the following standard way [6]:

$$
\begin{aligned}
S= & \frac{\beta N}{4} \sum_{\tilde{x}} \phi_{\tilde{x}}^{2}+\sum_{i=1}^{N / 2}\left(\sum_{x, y} \bar{\chi}_{x}^{i} M_{x, y} \chi_{y}^{i}\right. \\
& \left.+\frac{1}{8} \sum_{x} \bar{\chi}_{x}^{i} \chi_{x}^{i} \sum_{\langle\tilde{x}, x\rangle} \phi_{\tilde{x}}\right) .
\end{aligned}
$$

\subsection{The FSS results}

In order to study the critical behavior on lattices available to us we use the finite size scaling (FSS) method. Unlike previous bulk measurements of the critical exponents, when one has to keep the correlation length much smaller than the size of the box, in the FSS we let the correlation length saturate at the box size. This allows us to reach the Ginzburg region.

The results of the FSS analysis for $L_{t}=6$ lattices are summarized in Table 1. We see that all measured exponents show that the scaling very close to criticality is that of the Ising model in 2 dimensions rather than the MF one.

\subsection{The crossover exponent $x$}

A straightforward way to find the value of $x$ is to study the dependence of the order param- 
Table 1

Summary of FSS results and comparison with Ising model and MF scaling behavior.

\begin{tabular}{|l|lll|}
\hline Exponents & FSS & Z $(2)$ & MF \\
\hline$\nu$ & $1.00(3)$ & 1 & 0.5 \\
$\beta_{m} / \nu$ & $0.12(6)$ & 0.125 & 1 \\
$\gamma / \nu$ & $1.66(9)$ & 1.75 & 2 \\
\hline
\end{tabular}

eter, $\Sigma$, on $\beta$. Since $\Sigma$ vanishes at the critical point, it can be thought of as a measure of the distance from the criticality. We expect that for sufficiently small $\Sigma$, i.e., close to $\beta_{c}$, this dependence should be given by a power-law scaling of the 2 d Ising model: $\Sigma \sim(\text { const }-\beta)^{1 / 8}$. For larger $\Sigma$, further away from the criticality, the MF scaling holds: $\Sigma \sim\left(\text { const }^{\prime}-\beta\right)^{1 / 2}$. For even larger $\Sigma$ we should see the scaling corresponding to the fixed point of the $3 \mathrm{~d}$ Gross-Neveu model [6]: $\Sigma \sim\left(\text { const }^{\prime \prime}-\beta\right)^{1}$. In our simulations we can clearly resolve the MF region with the crossover towards the 2d-Ising region (Figure 1).

In order to find the exponent $x$ we use the relation: $\xi=1 /(2 \Sigma)$ valid in the MF region. Using the histogram reweighting method and monitoring the quality of the linear fit as we add or subtract points we find for the boundary of the MF region: $\Sigma_{\mathrm{MF}}=0.377(11)$, for $N=4, \Sigma_{\mathrm{MF}}=$ $0.213(4)$ for $N=12$, and $\Sigma_{\mathrm{MF}}=0.168(20)$ for $N=24$. It is clear that the non-trivial 2 dIsing region is squeezed as $N$ increases. The fit $\Sigma_{\mathrm{MF}}=$ const $\cdot N^{-x}$ gives $x=0.51(3)$ which is in agreement with the analytical prediction $x=0.5$ of eq. (3).

\section{Discussion and conclusions}

We have shown that a suppression of the critical region occurs in theories with large number of fermion species. The corresponding crossover exponent $x$ can be also determined (3).

The role of the fermions is to screen the effective self-coupling of the scalar field, $\lambda$. The strength of this effect depends on two factors: (i) large $N$, and (ii) large window of scales between the cutoff of the effective theory, $\Lambda$, and the temperature, $T$. In QCD there is almost an order of magnitude window between the scale of the

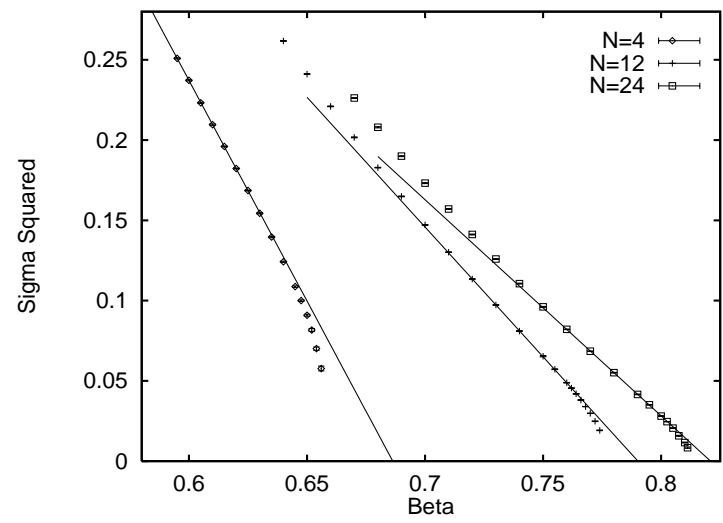

Figure 1. Order parameter squared vs. $\beta$ for lattice theories with $N=4,12,24\left(L_{t}=6\right)$. The straight lines are the fits to the data in the MF regions.

spontaneous symmetry breaking $\Lambda \sim 1 \mathrm{GeV}$, and $T_{c} \approx 160 \mathrm{MeV}$, which is presumably sufficient to drive the effective self-coupling of the scalar field to its infrared fixed point value at the scale of $T_{c}$. How small this value is then depends on the number of the fermions (the condition (i)). The value $N_{c}=3$, though not very large, can be considered large in some cases. One can expect, therefore, that in QCD the width of the critical region, where actual $\mathrm{O}(4)$ exponents are observed is suppressed. Further analysis is required to make a quantitative prediction.

\section{REFERENCES}

1. R. Pisarski and F. Wilczek, Phys. Rev. D29 (1984) 338; K. Rajagopal and F. Wilczek, Nucl. Phys. B399 (1993) 395.

2. M.A. Stephanov, Phys. Rev. D52 (1995) 3746.

3. B. Rosenstein, A.D. Speliotopoulos, and H.L. Yu, Phys. Rev. D49 (1994) 6822.

4. A. Kocic and J.Kogut, Phys. Rev. Lett. 74 (1995) 3109, Nucl. Phys. B455 (1995) 229.

5. J.B. Kogut, M.A. Stephanov, and C.G. Strouthos, hep-lat/9805023.

6. S. Hands, A. Kocic, and J.B. Kogut, Ann. Phys. 224 (1993) 29. 